\title{
Modeling of Triangular Lattice Space Structures with Curved Battens
}

\author{
Tzikang Chen ${ }^{*}$ \\ Vehicle Technology Directorate, Army Research Laboratory \\ NASA Langley Research Center, Hampton VA 23681 \\ and \\ John T. Wang ${ }^{\dagger}$ \\ NASA Langley Research Center, Hampton VA 23681
}

\begin{abstract}
Techniques for simulating an assembly process of lattice structures with curved battens were developed. The shape of the curved battens, the tension in the diagonals, and the compression in the battens were predicted for the assembled model. To be able to perform the assembly simulation, a cable-pulley element was implemented, and geometrically nonlinear finite element analyses were performed. Three types of finite element models were created from assembled lattice structures for studying the effects of design and modeling variations on the load carrying capability. Discrepancies in the predictions from these models were discussed. The effects of diagonal constraint failure were also studied.
\end{abstract}

\section{Introduction}

Lightweight lattice structures are being considered for many future space structure applications such as supporting booms for antennas, telescopes, and solar sails. For example, the triangular lattice beam column [1,2] for solar sail masts shown in Figure 1 is being developed under the support of the NASA In-space Propulsion Technologies Program. The triangular lattice beam-column contains very flexible components such as longerons, battens and diagonals. Longerons are the main load carrying members. Battens are used to keep the longerons in position and maintain the cross-sectional shape. Diagonals are tension members used to enhance the shear capability of the lattice beam. This type of lattice structure can be twisted and compressed (coiled) to a very small packing volume. The stored elastic energy (strain energy) of the coiled structure can be used for the deployment in space.

For a lattice structure that can be twisted and compressed, the battens need to maintain their curvature after assembly $[1,2]$. A batten's curvature is maintained by the compression forces that are induced by tensioning the diagonals. Therefore, an assembled lattice structure has a residual stress state. To predict accurately the residual stress state and shape, techniques that model the assembly process need to be developed.

Triangular lattice structures have been analyzed by many researchers [3-6]. Analytical solutions for investigating the effects of the local and global imperfections of longerons on the axial compressive strength of the lattice columns were performed by Crawford and Hedgepeth [3], Mikulas [4], and Crawford and Benton [5]. The finite element (FE) method and the continuum method have also been widely used for analyzing this type of structure, as summarized by Noor [6]. In the analyses in Refs. [3-6], the lattice structures were assumed to be assembled without residual stresses, and the battens were assumed to be straight. To the authors' knowledge, there are no published analysis results related to simulating the assembly process to account for the residual stress state in the assembled structure and to model the curvatures of the battens.

\footnotetext{
* Mechanical Engineer, Computational Structures and Materials Branch, MS 155

$\dagger$ Aerospace Engineer, Computational Structures and Materials Branch, MS 155, AIAA Senior Member
} 
The objectives of this paper are to develop modeling techniques that can simulate the assembly process of lattice structures, and to evaluate the effects of batten curvatures, residual stresses, and diagonal constraints on the load carrying capabilities of the assembled lattice structures. In order to simulate the assembly process of the lattice structures, a cable-pulley element developed in Ref [7] was implemented in ABAQUS [8]. This cable-pulley element can model a continuous cable passing through a pulley. The tension forces in the diagonals, the compression forces in the battens, and the curvatures of the battens in the equilibrium state of an assembled lattice structure can be determined by performing geometrically nonlinear analyses to simulate the assembly process.

In order to evaluate the effects of the batten curvatures, the residual stress, and the constraints of the diagonals on the load carrying capabilities of lattice structures, three types of models based on the assembled lattice structures were developed and analyzed. Models containing curved or straight battens and continuous or discontinuous diagonals were subjected to a transverse shear load with cantilever boundary conditions. The discrepancies among the predictions obtained from the various modeling approaches are discussed. Findings from this study can be used as guidance for developing proper modeling techniques for self-deployable lattice structures.

\section{Modeling of the assembly process}

To be able to predict the geometric shape and the residual stress states of an assembled lattice structure with curved battens such as the one shown in Figure 2(a), techniques for accurately modeling the assembly process need to be developed. The assembly process was simulated using a geometrically nonlinear analysis to obtain the equilibrium conditions of an assembled lattice structure. Note that three views and the dimensions of the lattice structure are shown in Figure 2(b).

The two dimensional (2-D) lattice panel shown in Figure 3, which represents one side of a short three dimensional (3-D) triangle lattice structure, was used to illustrate the assembly process and the simulation techniques. Similar assembly procedures and simulation techniques can be used for the triangular lattice structure shown in Figure 2(a) and are not presented in this paper for brevity. The dimensions of the lattice panel are shown in Figure 4. Note that after assembly, the lattice structures are in their equilibrium states with their diagonals tensioned to maintain the shape of the curved battens.

\section{Assembly process for a 2-D lattice panel}

The steps of the proposed assembly process are shown in Figure 3. The length of an unstrained straight batten is, $w+\delta w=346 \mathrm{~mm}$, which is longer than the final width, $w$, of the panel. The final width is, $w=341 \mathrm{~mm}$. The shortening $\delta w$ depends upon the curvature of the assembled batten. In this study, $\delta w$ is approximately $1.4 \%$ of the width of the panel. In the present models, the battens are attached to the longerons using pinned connectors that release the bending moments between the battens and longerons. The longerons are continuous rods. The top and bottom of the longerons are rigidly connected to other structural components, such as end plates or stiff battens. The assembly sequence for the 2-D lattice panel shown in Figure 3(a) is as follows:

1. In the first assembly step as shown in Figure 3(b), the straight battens and the longerons are joined together with the pinned connectors. The distance between the two longerons is larger than the designed width. The top and bottom of the longerons are not connected to other structural components in this step. The cables used as diagonals are linked from the top to the bottom on the longerons through the pulleys at the battenlongeron joints. The upper ends of the two cables are constrained to the top of the longerons, and a tension force is applied to the lower ends of the cables.

2. In the second assembly step as shown in Figure 3(c), a small transverse force, shown as a small arrow, is applied at the center of each batten to cause the battens to bow, while the two longerons are pushed toward each other. Once the battens begin to bow, the transverse force is gradually reduced. In this step, the tension forces in the cables are kept constant.

3. In the third assembly step as shown in Figure 3(d), the top and bottom of the longerons are attached to other stiff structural components, and the lower ends of the cables are fixed to the longerons after the distance between the longerons matches the designed width. Finally, the transverse longeron forces are released, and 
some small adjustments of the cable tension are performed to correct the minor width changes for ensuring the straightness of the longerons.

\section{ABAQUS simulation of the assembly process for the 2-D lattice panel}

The assembly process of the 2-D lattice panel shown in Figure 3, including longerons, battens, and diagonals, was simulated with the ABAQUS finite element (FE) code and a special user-defined cable-pulley element (see the Appendix for details). The material properties of the panel can be found in Table 1. Each longeron section between two battens is modeled with five beam elements, and each batten is modeled with six beam elements. The battens are pinned to the longerons. The connections between the longerons and battens are modeled with multi-point constraints (MPCs) that constrain the three translational displacements to be the same and allow the rotations to be free. The pinned connectors and pulleys are collocated at the batten-longeron joints shown as circles in Figure 3. The arrangement of the cable-pulley elements for the assembled 2-D lattice panel is shown in Figure 4(a). A typical cable-pulley element shown in Figure 4(a) contains two end nodes (nodes A and C indicated by " $x$ " marks) of the cable and one node at the pulley (node B indicated by a solid circle). The first (topmost) cable-pulley element of each cable was tied to the top of the longeron. The cable-pulley element at the bottom has a free end. In the assembly process, a tension force is applied to the free end of the cable to keep it taut.

Three solution steps used in the ABAQUS simulation follow:

1. The first step models the assembly process in Figures 3(b). The top and bottom components of the structure are removed from the initial FE model using ABAQUS “*MODEL CHANGE” keyword. All six Degrees of Freedom (DOFs) of the elements representing the longerons are constrained. A small tension force $(0.01 \mathrm{~N})$ is applied at the free end of the last cable element of each of the two cables. The "STABILIZE" option is used to eliminate the numerical singularities of the FE model in an unstressed state and to get the nonlinear analysis procedure started. To remove any residual forces induced by "STABILIZE", a dummy step is performed.

2. The second step consists of the assembly process shown in Figure 3(c). A small transverse force (5 N) is applied at the center of each batten at the beginning of the step. During this step, each of the two longerons is moved toward each other in the x-direction by $2.5 \mathrm{~mm}$ while the other five DOFs of the longeron nodes are fixed. The transverse force at the center of each batten is gradually removed while the tension force in the cable is kept constant.

3. The third step consists of the assembly process shown in Figure 3(d). In this step, the top and bottom structural components are added by using the "STRAIN FREE" option with the ABAQUS "*MODEL CHANGE" keyword. The free ends of the cables are fixed at the bottom of the longerons. Finally, all of the constraints on the longerons in the previous steps are released, and the structure reaches an equilibrium state.

The equilibrium shape of the lattice was examined. If the mid-width of the structure had any minor deviation from the designed width after the release of the transverse longeron forces, this deviation was corrected by adjusting the tension forces of the cables to ensure the straightness of the longerons. For the assembled model shown in Figure 3 (a), the vertical displacement at the midpoint of the batten is about $26 \mathrm{~mm}$, and the tension force in the cable is about $3.6 \mathrm{~N}$.

The simulation of the assembly process of the 3-D triangular lattice structure is similar to that of the 2-D lattice panel, except that in the second step, the three longerons of the 3-D lattice structure are moved toward the center of the triangle by $2.5 \mathrm{~mm}$. Because of the similarity, the ABAQUS simulation of the assembly process of the 3-D lattice structure is not presented in this paper.

\section{Three Types of Lattice Structure Models}

Three types of FE models were developed to investigate the effects of the curvature of the batten, the residual stress state, and the constraints of the diagonal cable on the load carrying capabilities of the lattice structure. The first type of the FE model is named the BCDC model, in which the battens are curved and the diagonals are continuous and tensioned. The 2-D assembled lattice panel model shown in Figure 4 contains compressed curved 
battens, nearly straight longerons, and tensioned continuous diagonals. The batten-longeron joint has a low-friction rail for the diagonal cable that can be approximated by a frictionless pulley. Therefore, the continuous diagonals can be modeled with frictionless cable-pulley elements.

The second type of the FE model is called the BCDD model, in which the battens are curved and the diagonals are discontinuous. The BCDD model has the same geometric shape as the assembled BCDC model, but the model does not have cable-pulley elements. The diagonals are modeled by truss elements with no compression modulus. The diagonal cables are fixed (glued) at every batten-longeron joint. Since the diagonal cables of typical deployable lattice space structures are fixed at the joints, this type of the model is considered to be the most accurate one for modeling the actual space lattice structure.

Note that the cable-pulley element can also be used to model a diagonal constraint failure. For example, the BCDC model can be considered as a failed BCDD model. In this case, all the diagonal constraints in the BCDD model are failed, and each of the unconstrained diagonals, that can pass through a joint, is modeled with a cablepulley element.

The third type of the FE model is called the BSDD models, in which battens are straight and diagonals are discontinuous. At the design and development stage, the batten curvature information may not be available to the structural analysts. Therefore, the battens are often modeled as straight beams or trusses instead of curved beams and the tension in the diagonals is neglected. The BSDD model is used to determine the effect of the curved battens. For this model, the assembly process isn't simulated, and the length of the battens is the same as the width of the assembled BCDD model. In the BSDD models, the battens are modeled with six beam elements, and the diagonals are modeled with truss elements without a compression modulus.

\section{Bending Analyses of Cantilevered Lattice Structures}

For the 2-D lattice panel and the 3-D triangular lattice structure, all three types of FE models described above were created and subjected to a transverse shear load with cantilever boundary conditions. The deflections and deformation patterns of different types of models are compared. The results show that the loading capabilities of lattice structures can be significantly affected by design and modeling variations.

\section{In-plane bending of the 2-D Lattice Panel}

The dimensions of the assembled 2-D panel are shown in Figure 4 and Table 2. The panel is composed of five bays. The assembled dimensions of all the models are the same except that the BSDD model has straight battens. The BCDC, BCDD, and BSDD 2-D lattice panel models are subjected to a transverse displacement (u) at the right top corner and clamped boundary conditions at the lower end, as shown in Figures 4-6.

The deformed shapes of the three lattice panel models are also shown in Figures 4-6. The deformed shape of the BCDC model shown in Figure 4 is dominated by shear deformation of the bays. The top of the structure remains nearly parallel to the bottom. The transverse force induces different tensions in the two cables. However, the tension in each of the two cables remains constant along their length due to the use of cable-pulley elements. The curvature of the battens increases a little with the deformation. No local buckling of longerons was observed.

The deformed shape of the BCDD model is shown in Figure 5. The deformation is also dominated by shear deformation, and the top remains nearly parallel to the bottom. Truss elements without a compression modulus are used for modeling the diagonals. No local buckling of longerons occurs. However, the batten curvatures in Figure 5(b) are significantly larger than those in Figure 5(a). The vertical displacement at the midpoint of the lowest batten is nearly doubled with the deformation.

The deformed shape of the BSDD model is shown in Figure 6. The bending deformation involves local buckling of the longeron subjected to compression. The deformed shape indicates that there is little shear deformation in each bay. The straight battens and discontinuous diagonals increase the shear stiffness substantially.

The load deflection curves for the three lattice panel models are shown in Figure 7. The transverse load carrying capability of the BCDC model is very low. The deformation of the BCDC model is mainly shear deformation. In 
pure shear deformation, a rectangular lattice bay becomes a parallelogram, where one diagonal is stretched and the other is shortened. The stretched diagonal in a lattice bay develops a tension force to resist the shear deformation. Since the cable diagonal cannot carry compression loads, only the tensioned diagonals contribute to shear stiffness of the structure. By using continuous diagonals, the cable can run through the pulley into the adjacent bays and the tension force cannot be developed. Therefore, the use of continuous diagonals in BCDC model results in little resistance to shear deformations.

The BCDD model can carry a slightly higher load than the BCDC model, as shown in Figure 7. After the maximum load is reached, the BCDD model load carrying capability is reduced. The significant increase in batten curvature can result in bay-shape changes causing the reduction of the shear resistance capability, as shown in Figure 5. The deformation of the battens also reduces the width of the lattice panel that may cause a reduction in the panel's in-plane bending rigidity.

The BSDD model with straight battens has the highest load carrying capability. The straight battens and the discontinuous diagonals prevent the development of shear deformations and also provide stiffer supports to the longerons to increase the bngeron's critical buckling load. The tip deflection is dominated by the bending deformation since the bays do not deform in shear. The bending moment of the structure induces compression loads in a longeron to cause local buckling. After the longeron buckles, the bending rigidity of the structure is reduced, but the loading capability is continuously increased, as shown in Figure 7.

\section{Bending of the 3-D Triangular Lattice Structure}

The dimensions of the 20-bay assembled 3-D triangular lattice shown in Figure 8 can be found in Figure 2(b) and Table 2. The material properties are listed in Table 1. The BCDC, BCDD, and BSDD models are used in the study. These models are subjected to an applied transverse tip-displacement at one end of the structure, and the other end is fixed.

The deformed shape of the BCDC model (curved battens and continuous diagonals) is shown in Figure 8(b) with a tip deflection of $302.5 \mathrm{~mm}$. The battens of the BCDC model remain nearly parallel to each other during deformation. This aspect indicates that the deformation is dominated by the shear deformation that is caused by the use of the continuous diagonals as discussed in the previous 2-D lattice panel section.

The deformed shape of the BCDD model (curved battens and discontinuous diagonals) is shown in Figure 9(b) with a tip deflection of $123.3 \mathrm{~mm}$. The compressed lower longeron buckles near the fixed end of the lattice structure. The dominant deformation mode of the structure is bending, which is different from the 2-D BCDD lattice panel, where the main deformation mode of the 2-D model is shear. The difference in deformation modes is due to the support that the batten arrangement provides, as opposed to the support provided by the curved battens in the 2D model. The stronger longeron support prevents the shear deformation. An additional analysis of the 2-D BCDD model was conducted to confirm this hypothesis. In the analysis, the modulus of the battens was doubled to provide stronger support to the longeron. Indeed, the lattice panel deforms in bending with local buckling similar to those of the 3-D model.

The deformed shape of the BSDD model (straight battens and discontinuous diagonals) with a tip deflection of $354.7 \mathrm{~mm}$ is shown in Figure 10(b). The dominant deformation mode of the structure is bending. The compressed lower longeron buckles near the fixed end of the lattice structure.

The load-deflection curves of all three triangular lattice structures are shown in Figure 11. The BCDC model has very little capability for carrying the transverse shear load, which is similar to the 2-D BCDC model. Under shear deformation, the shapes of the bays change, and the bending rigidity of the structure is substantially reduced.

Since the BCDD model deforms in bending, as shown in Figure 9(b), the BCDD model's load carrying capability is greater than the BCDC model. The local buckling of the compressed lower longeron occurs at Point A in Figure 11. After the local buckling, the bending rigidity is greatly reduced, and the structure can carry little additional load. Numerical difficulties are encountered after the tip deflection reaches about $120 \mathrm{~mm}$, and converged solutions cannot be obtained. 
The buckling load of the BSDD model (Point B) is greater than that of BCDD model (Point A) as shown in Figure 11. This increased buckling strength is due to the greater support provided to the longerons by the straight battens, as opposed to that provided by the curved battens. The model with straight battens has the highest load carrying capability. After buckling of the lower longeron, the loading capability of the BSDD model continues to increase. Since the BCDD model includes all the structural details of a typical deployable triangular lattice structure, it is the most accurate structural model. Thus, using the conventional engineering BSDD model may result in an over-prediction of the load carrying capability of an actual lattice structure design.

One important issue that needs to be studied consists of the effects of the failures or defects in space lattice structures. As mentioned previously, the BCDD model becomes a BCDC model when all diagonals are not fixed to the batten-longeron joints and are allowed to pass through the joints. Such loss of constraints may be caused by failures. By examining the load-deflection curves of both models in Figure 11, we can conclude that this type of failure or manufacturing defect can have a detrimental effect on the load carrying capability of the triangular lattice structure. This is an extreme failure case that can be used to predict a lower bound to the load carrying capability of the structure. Other failure cases with partial failure of diagonal constraints in the BCDD model can be investigated in the future.

\section{Concluding remarks}

Modeling techniques were developed for simulating the assembly process of the lattice structures so that the assembled shape of the curved battens, the tension in the diagonals, and the compression in the battens can be predicted for the assembled model. To be able to perform the assembly simulation, a cable-pulley element was successfully implemented in ABAQUS as a user defined element, and geometrically nonlinear finite element analyses were performed.

Three types of finite element models, denoted the BCDC, BCDD and BSDD models, were created of the assembled 2-D lattice panel and 3-D triangular lattice structures to study the effects of the design and modeling variations on the load carrying capabilities of each configuration. The assembled BCDC model consists of curved battens and continuous cable diagonals. The assembled BCDD model consists of curved battens and discontinuous cable diagonals. The BCDD model represents an actual space lattice structure in which the battens are curved and the continuous cables are fixed to every joint along the longerons to reduce shear deformations. The assembled BSDD model consists of straight battens and discontinuous cable diagonals. The BSDD model is a modeling variation of the BCDD model in which the curvature of the battens and the residual tension in the diagonals are neglected.

These three types of lattice models with cantilever boundary conditions were subjected to a transverse shear load. Load-deflection curves show that the BCDC lattice undergoes a large shear deformation and has a small load carrying capability. The BCDD lattice has a higher load carrying capability, and the BSDD lattice has the highest load carrying capability. Since the BCDD model includes all the structural details of a typical deployable triangular lattice structure, it is the most accurate structural model. Thus, using the conventional engineering BSDD model may result in an over-prediction of the load carrying capability of an actual lattice structure design.

The cable-pulley element can also be used for investigating the effects of defects in lattice structures. As discussed in this paper, the BCDC model is an extreme case of diagonal constraint failure that can be used to predict a lower bound to the load carrying capability of typical lattice structures.

\section{Acknowledgements}

The work described in this paper was funded in part by the In-Space Propulsion Technology Program, which is managed by NASA's Science Mission Directorate in Washington, D.C., and implemented by the In-Space Propulsion Technology Office at Marshall Space Flight Center in Huntsville, AL. The program objective is to develop in-space propulsion technologies that can enable or benefit near and mid-term NASA space science missions by significantly reducing cost, mass or travel times. 


\section{References}

${ }^{1}$ Murphy, D., Trautt, T., McEachen, M., Messner, D., Laue G., and Gierow, P., "Progress and Plans for System Demonstration of a Scalable Square Solar Sail," 14th AAS/AIAA Space Flight Mechanics Conference, Maui, Hawaii, February 8-12, 2004.

${ }^{2}$ Murphy, D. M., Macy, B. D., and Gaspar, J. L., "Demonstration of a 10-m Solar Sail System," AIAA 20041576, 45th AIAA/ASME/ASCE/AHS/ASC Structures, Structural Dynamics \& Materials Conference, Palm Springs, California, April 19 - 22, 2004.

${ }^{3}$ Crawford, R. F., and Hedgepeth, J. M., "Effects of Initial Waviness on the Strength and Design of Built-up Structures," AIAA Journal, 13(5), 1975, pp. 672-675.

${ }^{4}$ Mikulas, M. M., "Structural Efficiency of Long, Lightly-Loaded Truss and Isogrid Columns for Space Applications," NASA TM 78687, July 1978.

${ }^{5}$ Crawford, R. F. and Benton, M. D., "Strength of Initially Wavy Lattice Columns," AIAA Journal, 18(5), 1980, pp. 581-584.

${ }^{6}$ Noor, A. K., "Continuum Modeling for Repetitive Lattice Structures," Applied Mechanics Review, 41(7), 1998, pp. 285-296.

${ }^{7}$ Aufaure, M., “A Finite Element of Cable Passing through a Pulley," Computers \& Structures, 46(5), 1993, pp. 807-812.

${ }^{8}$ ABAQUS/Standard User's Manual, version 6.5, Hibbitt, Karlsson, and Sorensen, Inc., 2004.

${ }^{9}$ Cardona, A. and Geradin, M., "A Beam Finite Element Nonlinear Theory with Finite Rotations," International Journal for Numerical Methods in Engineering, 26, 1988, pp 2403-2438.

\section{APPENDIX. Cable-Pulley Element}

The cable and pulley mechanism is commonly used in mechanical and structural systems. A cable-pulley element was implemented in ABAQUS based on the Ref. [7]. Figure A1(a) shows the configuration of a cable with ends $\mathrm{N}_{1}$ and $\mathrm{N}_{2}$ passing through a pulley $\mathrm{N}_{3}$. Figure A1(b) shows the internal force of the element. To model large displacements of the cable and the pulley, a geometrically nonlinear finite element method is used.

The assumptions used for the cable-pulley element are:

1. The cable is perfectly flexible and can only carry tension force.

2. The radius of the pulley is zero.

3. The pulley is frictionless. The tension force in the cable segments is constant $\left(\left|\mathbf{F}_{1}\right|=\left|\mathbf{F}_{2}\right|\right)$. The equilibrium condition of the element is $\mathbf{F}_{3}=-\left(\mathbf{F}_{1}+\mathbf{F}_{2}\right)$.

4. Thermal effects are neglected.

5. The weight of the cable is neglected (the sag effect is not considered).

6. The cable-pulley element can be used to model large displacements for both the cable and the pulley.

7. The strain of the cable is assumed to be small (Poisson's effect of the cable is not considered).

\section{Formulation of the element}

With the aforementioned assumptions, the cable-pulley element can be idealized as shown in Figure A1(b). The two strands $\mathrm{N}_{1} \mathrm{~N}_{3}$ and $\mathrm{N}_{2} \mathrm{~N}_{3}$ remain rectilinear. The vectors of the strands can be written as

$$
\begin{aligned}
& \mathbf{L}_{1}=\mathbf{X}_{1}+\mathbf{U}_{1}-\left(\mathbf{X}_{3}+\mathbf{U}_{3}\right) \\
& \mathbf{L}_{2}=\mathbf{X}_{2}+\mathbf{U}_{2}-\left(\mathbf{X}_{3}+\mathbf{U}_{3}\right)
\end{aligned}
$$




$$
\begin{gathered}
\left|L_{1}\right|=\sqrt{\mathbf{L}_{1}^{T} \mathbf{L}_{1}} \quad, \quad\left|L_{1}\right|=\sqrt{\mathbf{L}_{2}^{T} \mathbf{L}_{2}} \\
\mathbf{l}_{1}=\mathbf{L}_{1} /\left|L_{1}\right|, \quad \mathbf{l}_{2}=\mathbf{L}_{2} /\left|L_{2}\right|
\end{gathered}
$$

where $\mathbf{X}_{i}, \mathbf{U}_{i}$ are the coordinates and the displacement vectors of the $i$-th node, respectively. $\mathbf{L}_{i}$ and $\mathbf{l}_{i}$ are the vector and the unit vector of the $i$-th cable strand, respectively. $\left|L_{i}\right|$ is the length of the $i$-th cable strand.

The cable is assumed to be linear elastic and the constitutive relation between the tension force $N$ and cable strain $\varepsilon$ can be expressed as

$$
N=\left\{\begin{array}{cc}
E A \varepsilon & \varepsilon \geq 0 \\
0 & \varepsilon<0
\end{array}\right.
$$

where $E$ and $A$ are the modulus and area of the cross section of the cable, respectively. The strain $\varepsilon$ must remain small, so that the cross-sectional area, $A$, of the cable is unchanged. The strain $\varepsilon$ is defined as

$$
\varepsilon=\left(\left|L_{1}\right|+\left|L_{2}\right|-\mid L_{0}\right) / \mid L_{0}
$$

where $|L|_{0}$ is the total unstrained length of the cable $|L|_{0}=\left|L_{1}\right|_{0}+\left|L_{2}\right|_{0}$.

The points $\mathrm{N}_{1}, \mathrm{~N}_{2}$ and $\mathrm{N}_{3}$ of Figure A1(b) are referred as nodes of the element, and the third node, $\mathrm{N}_{3}$, is at the location of the pulley. The internal forces are the forces that must be exerted on the nodes to keep it in its equilibrium configuration, as shown in Figure A1(b). These forces are defined as

$$
\mathbf{F}_{1}=N \cdot \mathbf{l}_{1}, \quad \mathbf{F}_{2}=N \cdot \mathbf{l}_{2}, \quad \mathbf{F}_{3}=-\left(\mathbf{F}_{1}+\mathbf{F}_{2}\right)
$$

The incremental nodal displacements and the incremental internal forces of the nonlinear cable element are defined as

$$
\delta \mathbf{U}=\left\{\begin{array}{l}
\delta \mathbf{U}_{1} \\
\delta \mathbf{U}_{2} \\
\delta \mathbf{U}_{3}
\end{array}\right\}, \quad \delta \mathbf{F}=\left\{\begin{array}{l}
\delta \mathbf{F}_{1} \\
\delta \mathbf{F}_{2} \\
\delta \mathbf{F}_{3}
\end{array}\right\}
$$

and they are related as

$$
\delta \mathbf{F}=\mathbf{K} \cdot \delta \mathbf{U}
$$

where $\mathbf{K}$ is the tangent stiffness matrix and $\delta \mathbf{F}$ can be calculated by [9],

$$
\delta \mathbf{F}=\lim _{? \rightarrow 0} \frac{d}{d \eta} \mathbf{F}(\mathbf{U}+\eta \cdot \delta \mathbf{U})
$$

The final expression of $\mathbf{K}$ is:

$$
\mathbf{K}=\left[\begin{array}{ccc}
\mathbf{K}_{11} & \mathbf{K}_{12} & -\left(\mathbf{K}_{11}+\mathbf{K}_{12}\right) \\
\mathbf{K}_{12}^{T} & \mathbf{K}_{22} & -\left(\mathbf{K}_{22}+\mathbf{K}_{12}^{T}\right) \\
-\left(\mathbf{K}_{11}+\mathbf{K}_{12}^{T}\right) & -\left(\mathbf{K}_{22}+\mathbf{K}_{12}\right) & -\left(\mathbf{K}_{13}+\mathbf{K}_{23}\right)
\end{array}\right]
$$

where: 


$$
\begin{gathered}
\mathbf{K}_{11}=\left(\frac{E A}{|L|_{0}}-\frac{N}{\left|L_{1}\right|}\right) \mathbf{l}_{1} \cdot \mathbf{l}_{1}^{T}+\frac{N}{\left|L_{1}\right|} \mathbf{I}_{3} \\
\mathbf{K}_{22}=\left(\frac{E A}{|L|_{0}}-\frac{N}{\left|L_{2}\right|}\right) \mathbf{l}_{2} \cdot \mathbf{l}_{2}^{T}+\frac{N}{\left|L_{2}\right|} \mathbf{I}_{3} \\
\mathbf{K}_{12}=\frac{E A}{|L|_{0}} \mathbf{l}_{1} \mathbf{l}_{2}^{T}
\end{gathered}
$$

where $\mathbf{I}_{\mathbf{3}}$ is a $3 \times 3$ identity matrix.

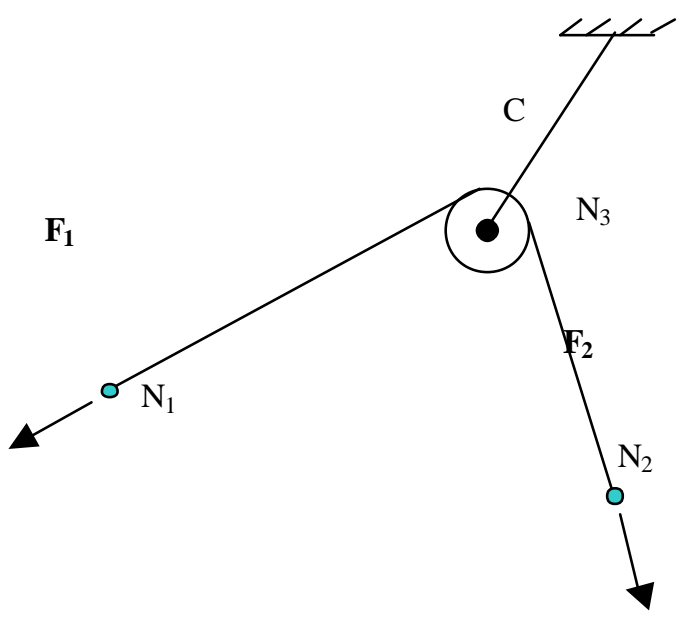

(a) Actual configuration

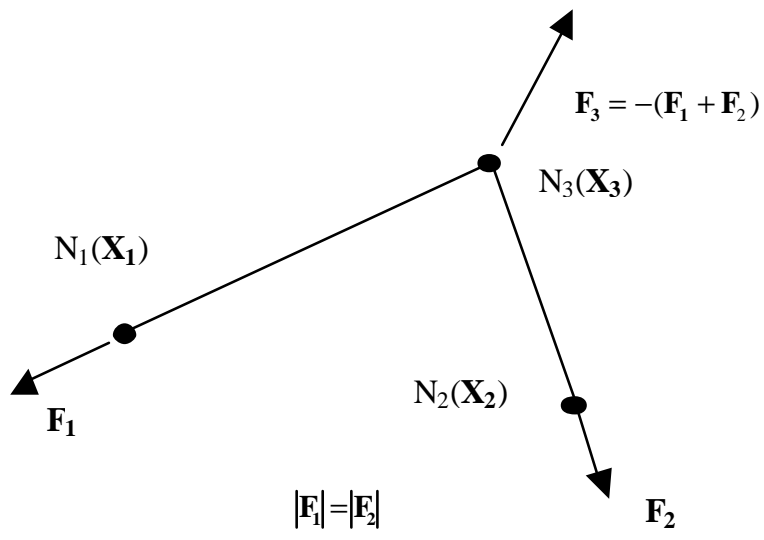

(b) Cable-pulley element

Figure A1. A cable passing through a pulley

Table 1. Material Properties

\begin{tabular}{|c|c|}
\hline $\begin{array}{c}\text { Component } \\
\text { name }\end{array}$ & $\begin{array}{c}\text { Young's modulus E } \\
\left(\mathrm{N} / \mathrm{mm}^{2}\right)\end{array}$ \\
\hline Longeron & $190.0 \times 10^{3}$ \\
\hline Diagonal & $120.0 \times 10^{3}$ \\
\hline Batten & $200.0 \times 10^{3}$ \\
\hline
\end{tabular}

Table 2. Cross-sectional Dimensions

\begin{tabular}{|c|c|c|}
\hline \multirow{2}{*}{$\begin{array}{c}\text { Component } \\
\text { name }\end{array}$} & \multicolumn{2}{|c|}{ Cross-sectional shape } \\
\cline { 2 - 3 } & Circular (Radius) & Rectangular \\
\hline Longeron & $1.23 \mathrm{~mm}$ & \\
\hline Diagonal & $0.37 \mathrm{~mm}$ & \\
\hline Batten & & $1.3 \mathrm{~mm} \times 1.5 \mathrm{~mm}$ \\
\hline
\end{tabular}




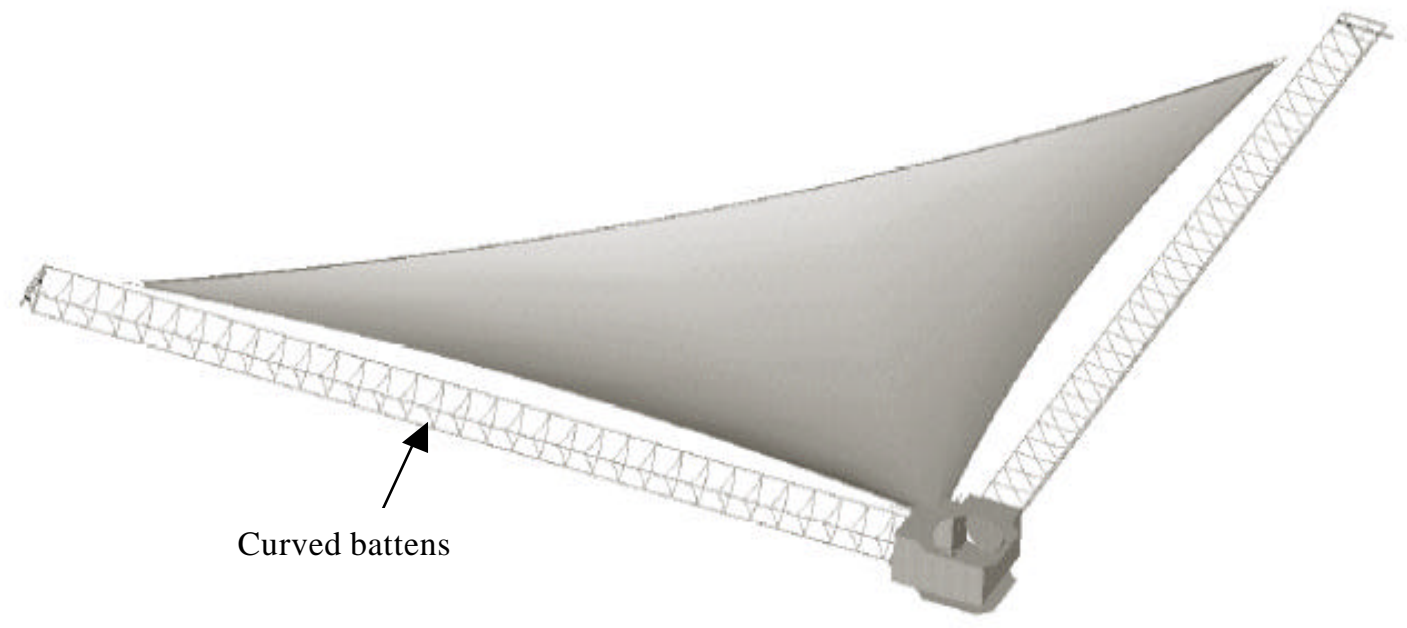

Figure 1. Depiction of a 10-m solar sail quadrant system [2]

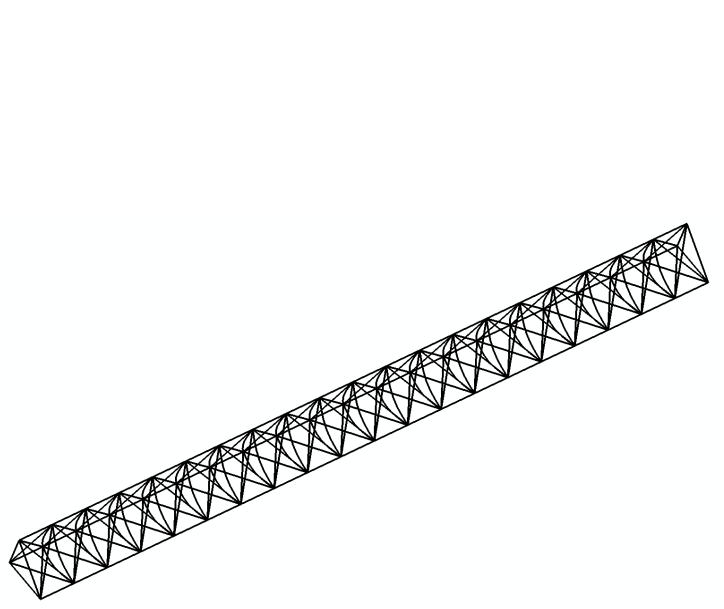

(a) Assembled triangular lattice structure

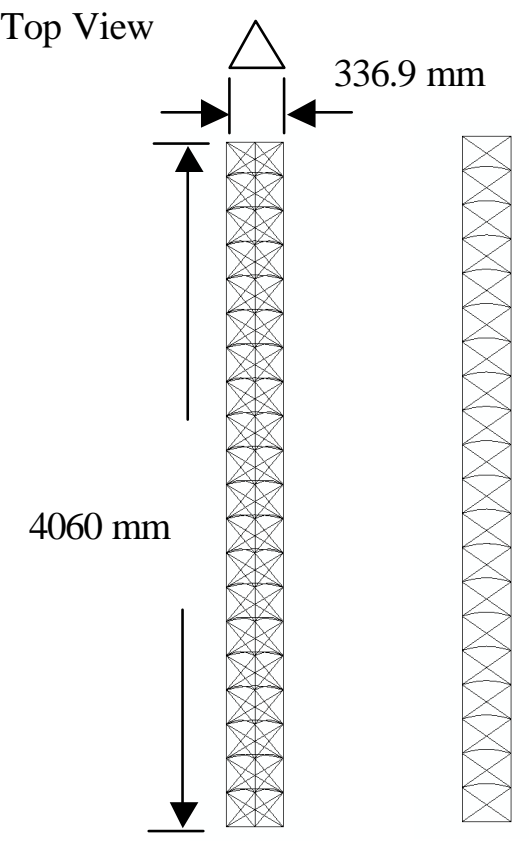

Front View Side View

(b) Different views

Figure 2. Dimensions of the assembled triangular lattice structure 


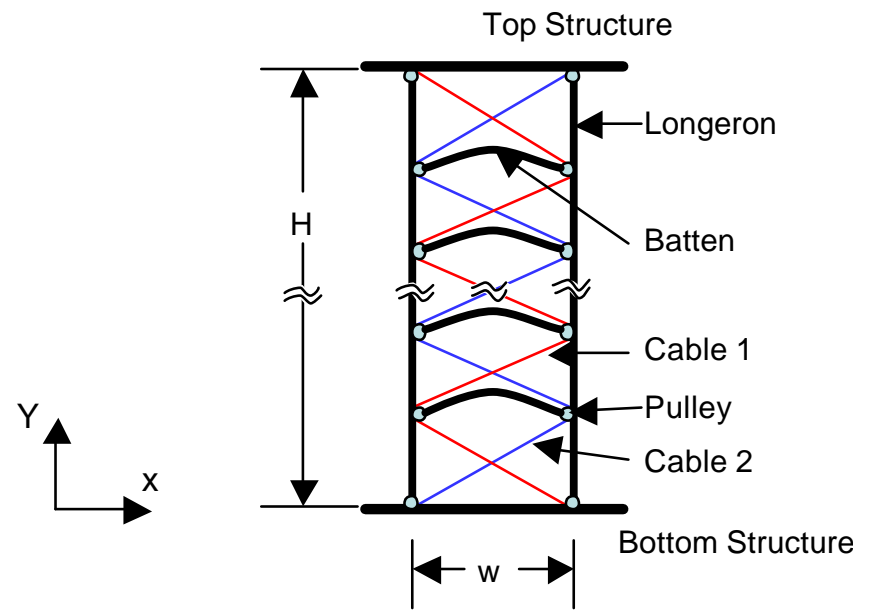

(a) Assembled lattice panel

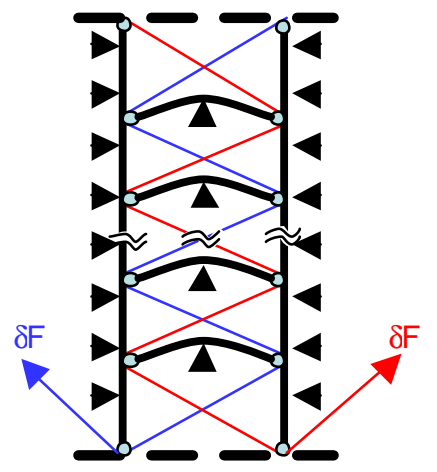

(c) Second assembly step panel

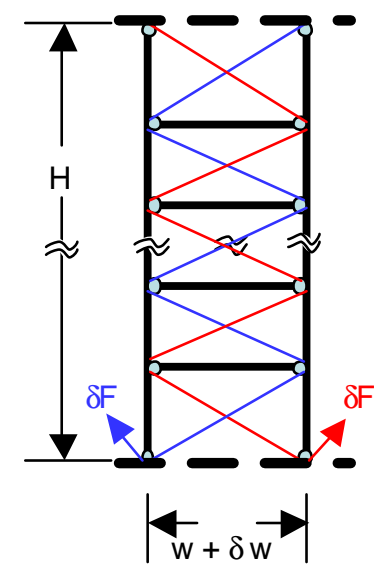

(b) First assembly step

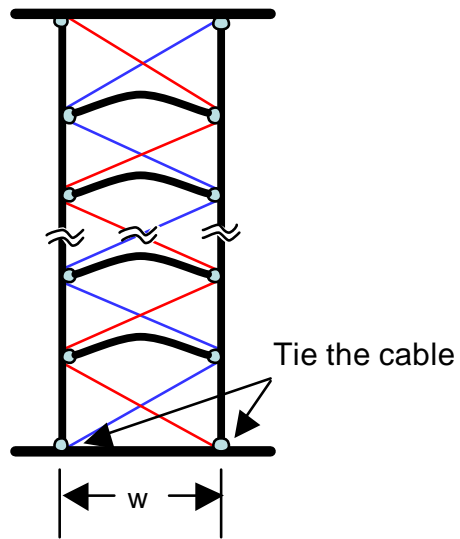

(d) Third assembly step

Figure 3. Lattice panel assembly procedure

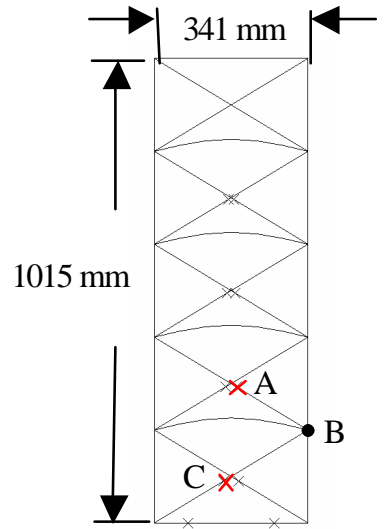

(a) Initial shape

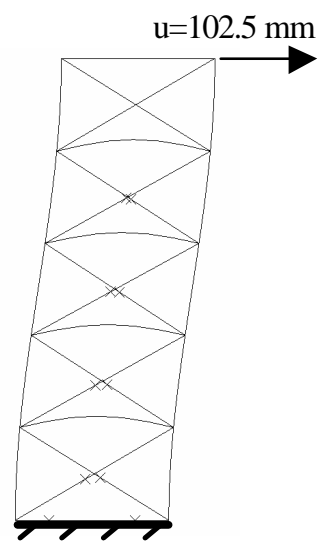

(b) Deformed shape
Figure 4. Lattice panel with curved battens and tensioned continuous diagonals (BCDC model)

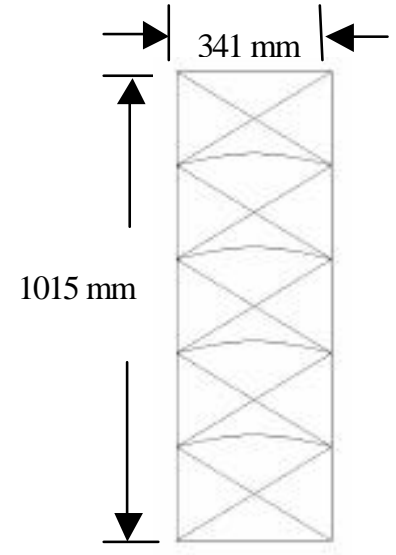

(a) Initial shape

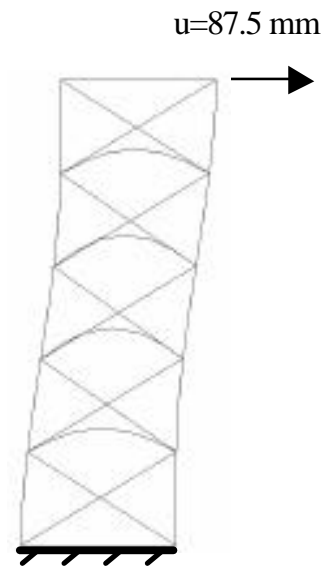

(b) Deformed shape

Figure 5. Lattice panel with curved battens and tensioned discontinuous diagonals (BCDD model) 


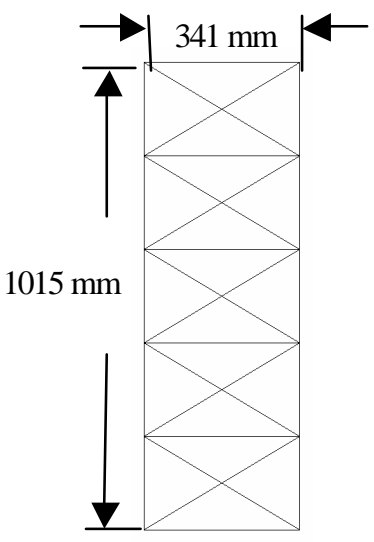

(a) Initial shape

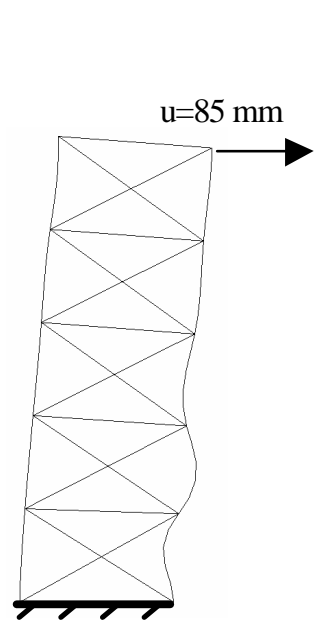

(b) Deformed shape

Figure 6. Lattice panel with straight battens and discontinuous diagonals (BSDD model)

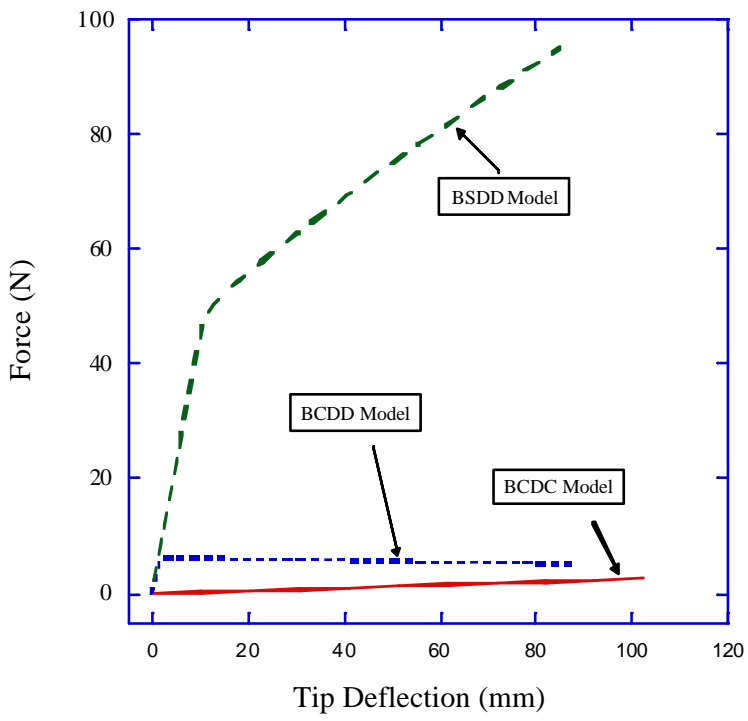

Figure 7. Transverse force versus tip deflection of cantilevered lattice panels

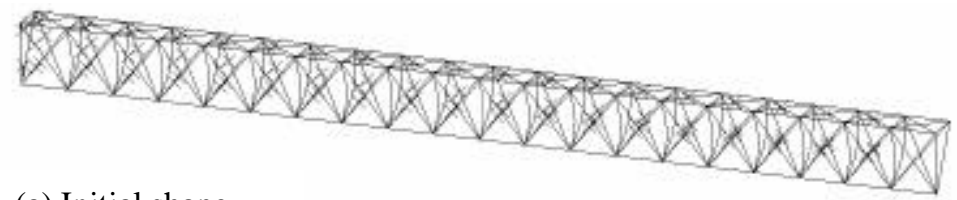

(a) Initial shape

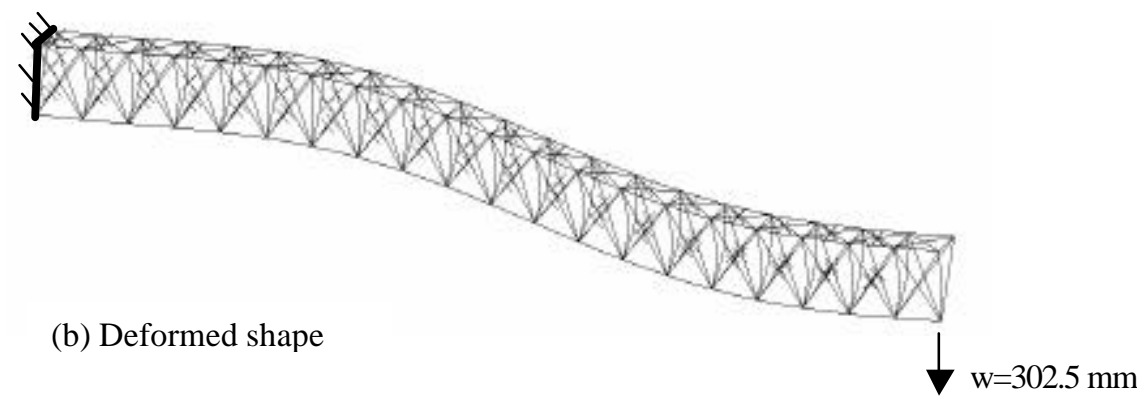

Figure 8. Triangular lattice structure with curved battens and tensioned continuous diagonals (BCDC model) 

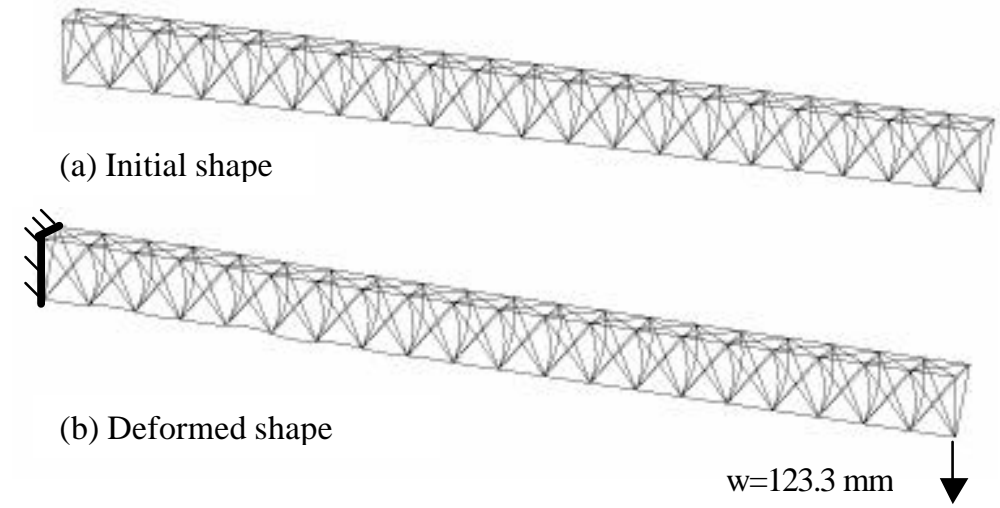

Figure 9. Triangular lattice structure with curved battens and tensioned discontinuous diagonals (BCDD model)
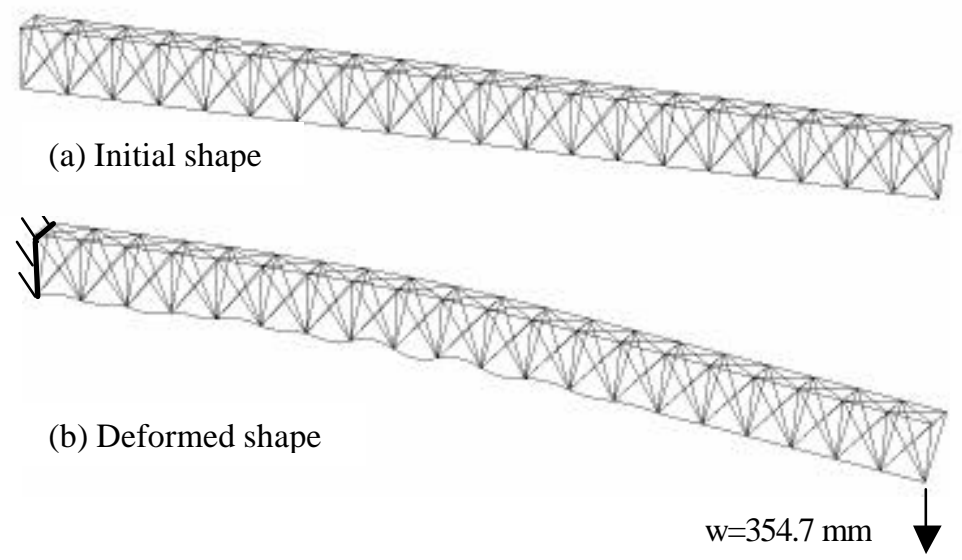

Figure 10. Triangular lattice structure with straight battens and discontinuous diagonals (BSDD model) 


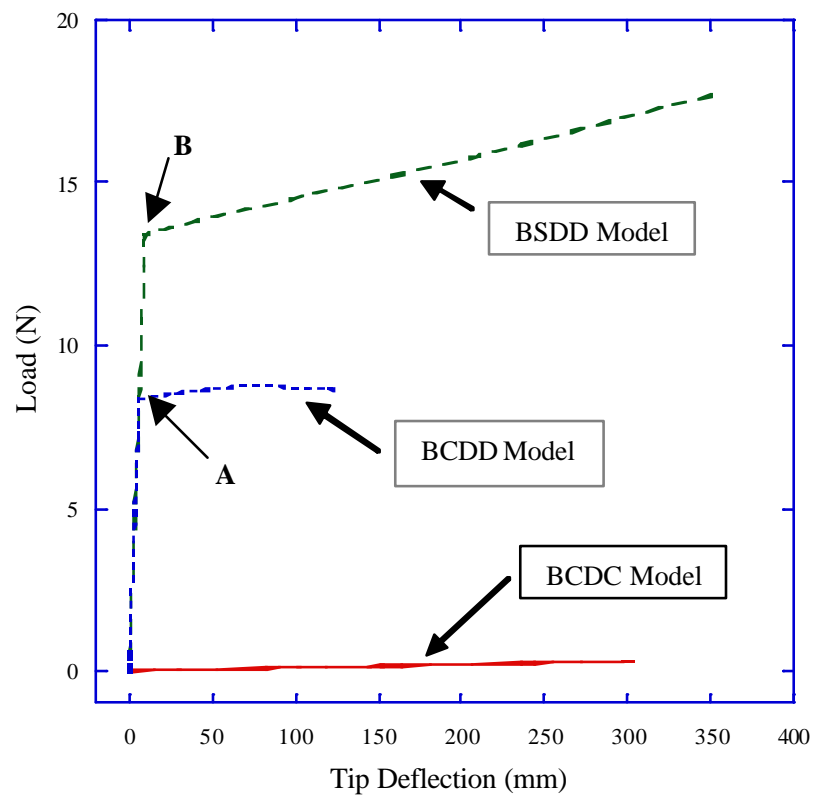

Figure 11. Transverse force versus tip deflection of cantilevered lattice panels 